\title{
The retrieval of antibiotic sensitivity by 2- phenoxyethanol adapted-multi drug resistant (MDR) clinical isolate of Pseudomonas aeruginosa
}

\author{
S. M. A. Abdelmalek ${ }^{1 *}$, S. Zeidan ${ }^{1}$ and Y. R. Badran ${ }^{2}$ \\ ${ }^{1}$ Petra University-Faculty of Pharmacy and medical Sciences, Jordan. \\ ${ }^{2}$ University of Jordan, Faculty of Medicine, Jordan.
}

Accepted 17 May, 2013

\begin{abstract}
2-Phenoxyethanol (2-PE) is a biocide used as a preservative in pharmaceutical, cosmetic and perfumery formulations. It is known for its rapid bactericidal effect against a wide range of bacteria. In the present study, clinical samples of multi drug resistant (MDR) Pseudomonas aeruginosa and Escherichia coli were grown in an increasing sub-minimal inhibitory concentration of 2-PE to observe if adaptation could be obtained and consequently retrieval of antibiotic susceptibility to clinically used antibiotics. Minimal inhibitory concentration (MIC) of 2-PE was determined for both MDR $E$. coli and $P$. aeruginosa. Adaptation to the biocide was attempted by repeated sub-culturing of the isolates in increasing sub-minimal-inhibitory concentration of the biocide. $E$. coli was able to grow in the presence of sub-MIC of 2-PE for two passages only and a slight rise in MIC was observed. MDR $P$. aeruginosa rapidly obtained an adaptive resistance towards the biocide with an increase in MIC from 5.4 to $13.7 \mathrm{~g} \%$. Antibiotic sensitivities for both, MDR and MDR-2-PE adapted $E$. coli and $P$. aeruginosa were detected. MDR2-PE resistant $P$. aeruginosa showed significant improvement in antibiotic sensitivity while no change was observed with $E$. coli. Our results suggest an intriguing species specific link between biocide resistance and changes in antibiotic susceptibilities, findings that could be utilized in controlling the spread of antibiotic resistance and ambitiously improving the activity of some existing antibiotics.
\end{abstract}

Key words: Pseudomonas aeruginosa, 2-phenoxyethanol, antibiotic resistance.

\section{INTRODUCTION}

The role of microbicides in emerging bacterial resistance towards antibiotics is still controversial. Several studies have pointed out that the increase in the use of biocides in domestic settings as well as hospital environments furnishes for the selection of antibiotic resistant strains (Aiello, 2005; Braoudaki and Hilton, 2004; Loughlin et al.,
2002; Walsh, 2003).On the other hand, adaptive resistance to some biocides has been reported by some researchers to reduce antibiotic resistance (Abdel Malek 2009; Joynson et al., 2002). Benzalkonium chloride (BC) adaptation for example, has been described not only for laboratory strains, but also for clinical isolates of

*Corresponding author. Email: mlrsam2001@yahoo.com. Fax 00962-6-5715570.

Abbreviations: BC, Benzalkonium chloride; MIC, minimum inhibitory concentration; 2-PE, 2-phenoxyethanol; MDR, multi drug resistant; DMF, dimethylformamide; NB, nutrient broth; MHB, Mueller Hinton Broth. 
Pseudomonas aeruginosa. Clinical isolates from cystic fibrosis patients were sub-cultured into increasing concentrations of benzalkonium chloride and adaptation to BC was accompanied by a decrease in minimum inhibitory concentration (MIC) to several antibiotics and accelerated the loss of resistance to imipenem (Joynson et al., 2002). BC was even suggested to be used in hospital hygiene to help overcome antibiotic resistance in clinical environment (Joynson et al., 2002). It is generally accepted that in contrast to chemotherapeutic agents, biocides have multiple target sites within the microbial cell and the overall damage to these target sites results in the bactericidal effect. However, the use of biocides at sub-lethal concentrations have been found to result in adaptive resistance to these biocides and cross resistance to antibiotics (Braoudaki and Hilton, 2004; Fraise, 2002; Hegstad et al., 2010; Karatzas et al., 2007). Since biocides have multiple mechanisms of action within the cell, adaptations to variable biocides by different microbes will result in variable alterations in antibiotic susceptibility, an issue that mandates further investigation into its aspects (Aiello et al., 2007).

E. coli $\mathrm{O} 157$ strains for example acquired high levels of resistance to triclosan after only two sub lethal exposures and when adapted they repeatedly demonstrated decreased susceptibilities to various antimicrobial agents, including chloramphenicol, erythromycin, imipenem, tetracycline, and trimethoprim, as well as to a number of biocides (Braoudaki and Hilton, 2004). Whereas $P$. aeruginosa had shown altered antibiotic susceptibility after adaptation to various biocides (Abdel Malek and Badran 2010; Walsh et al., 2003). Mechanisms of biocide resistance were summarized by Maillard (2010), in a recent review to be altering between changes in the outer membrane permeability, expression and/or over expression of multidrug efflux systems or alteration in the target site (Maillard, 2010). Antimicrobials can also act as triggers of the microbial stress response seen in drug resistance (Poole, 2012). Thus, by the same mechanism, stress response towards biocides could add to some microbes ability to resist antibiotics.

2-phenoxyethanol (2-PE), a powerful antimicrobial that is used as a preservative in pharmaceutical, cosmetic and perfumery formulations, has a rapid bactericidal effect against a wide range of bacteria (including $P$. aeruginosa) and has been used as a general antiseptic, bladder irrigant and in the treatment of wounds and burns (octenidine dihydrochloride) (Hubner et al., 2010; Wilson et al., 1990). 2-PE affects a multitude of intracellular targets (Beveridge et al., 1991; Denyer, 1995). At sub lethal biocide levels, a variety of concentration-dependent inhibitory processes take place, from actions such as potassium-proton antiporters and respiration uncouplers to competitive inhibition of $\mathrm{NADH}$ binding by malate dehydrogenase and slowed DNA biosynthesis relative to general anabolism(Russell, 2003).

Previous work by Abdelmalek and Badran (2010) had shown that 2-PE adaptation of $P$. aeruginosa PAO1 involved outer membrane alterations but did not involve resistance nodulation cell division (RND) efflux pumps (Abdelmalek and Badran, 2010), and was accompanied by a reduced resistance to some antibiotics. PAO1 $P$. aeruginosa is a laboratory strain that has been studied extensively in the field of biocide resistance (Kern et al., 1994; Maillard, 2010; Winder et al., 2000). It has been found to adapt to most biocides and this adaptation resulted from outer membrane protein alterations, fatty acid composition changes or efflux mechanisms (Abdel Malek et al., 2009; Abdelmalek and Badran, 2010; Jones et al., 1989; Strateva and Yordanov 2009). In this study, clinical isolates of MDR P. aeruginosa and Escherichia coli collected from immune compromised patients are subjected to sub inhibitory concentrations of 2-PE to investigate the possibility of adaptation to this biocide and whether antibiotic sensitivity will be affected.

\section{MATERIALS AND METHODS}

\section{Bacterial cultures}

Two multi drug resistant (MDR) isolates $E$. coli and $P$. aeruginosa were obtained from KHCC (King Hussein Cancer Center, Amman, Jordan) and were sub-cultured on nutrient agar and maintained by subculturing in Nutrient agar slopes. These cultures were used as the $2-\mathrm{PE}$-sensitive strains. Passaged cells were preserved at $-70^{\circ} \mathrm{C}$ in glycerol.

\section{Controls}

The following strains were used as controls: E. coli ATCC 25922 wild type, a kind gift from Specialty Hospital, Amman, Jordan. $P$. aeruginosa PAO1. (NCIMB 10548), obtained from National Collection of Industrial, Marine and Food bacteria (NCIMB) (Aberdeen,UK).

\section{Chemicals}

2-PE (Sigma-Aldrich) was dissolved in dimethylformamide (DMF), and variable concentrations were prepared in sterile distilled $\mathrm{H}_{2} \mathrm{O}$ and kept in amber glass bottles at $4^{\circ} \mathrm{C}$ until used within a week from preparation. Antibiotic discs of: Gentamicin (CN) $10 \mu \mathrm{g}$, amoxacillin (AML) $25 \mu \mathrm{g}$, doxycyclin (DO) $30 \mu \mathrm{g}$, amikacin (AK) $30 \mu \mathrm{g}$, rifampicin (RD) $5 \mu \mathrm{g}$, norfloxacin (NOR) $10 \mu \mathrm{g}$, vancomycin (VA) $30 \mu \mathrm{g}$, cefotaxime (CTX) $30 \mu \mathrm{g}$, cephalexin (CL) $30 \mu \mathrm{g}$, ofloxacin (OFX) 5 $\mu \mathrm{g}$, chloramphenicol (C) $30 \mu \mathrm{g}$, tobramycin (TOB) $10 \mu \mathrm{g}$, azithromycin (AZM) $15 \mu \mathrm{g}$, sulphamethoxazole (SXT) $25 \mu \mathrm{g}$, ticar/clav (TIM) $85 \mu \mathrm{g}$, ciprofloxacin (CIP) $5 \mu \mathrm{g}$, cefixim (CFM) $5 \mu \mathrm{g}$, minocyclin (MH) $30 \mu \mathrm{g}$, aztreonam (ATM) $30 \mu \mathrm{g}$, ceftizoxime (ZOX) 30 $\mu \mathrm{g}$, piperacillin (PRL) $100 \mu \mathrm{g}$, ceftazidime (CAZ) $30 \mu \mathrm{g}$, ampicillin (AMP) $10 \mu \mathrm{g}$, imipenem (IPM) $10 \mu \mathrm{g}$, nitrofuranton (F) $300 \mu \mathrm{g}$, were purchased from Oxoid (Germany).

\section{Methods}

Determination of MIC of 2-PE was done using the tube dilution method (Bloomfield, 1991). MICs were determined in Mueller Hinton Broth (MHB) medium $(9.0 \mathrm{ml})$ with an inoculum of $100 \mu \mathrm{L}$ from an overnight culture in the same medium grown at $37^{\circ} \mathrm{C}$ in a 
Table 1. Minimal Inhibitory Concentration (MIC) g\% of 2-PE for $E$. coli and $P$. aeruginosa strains.

\begin{tabular}{lc}
\hline Microbial strain & MIC g\% \\
\hline E. coli 25922 & 4.4 \\
MDR E. coli P0* & 4.4 \\
MDR E. coli P1 & 5.3 \\
MDR E. coli P2 & 5.3 \\
P. aeruginosa PAO1 & 3.3 \\
MDR P. aeruginosa P0 & 6.1 \\
MDR P. aeruginosa P1 & 6.8 \\
MDR P. aeruginosa P2 & 8.8 \\
MDR P. aeruginosa P3 & 11.3 \\
MDR P. aeruginosa P4 & 13.7 \\
\hline
\end{tabular}

${ }^{*} \mathrm{PN}$, Number of subcultures (passages) in a sub-minimal inhibitory concentration of 2-PE; 2-PE, 2- phenoxyethanol; MDR, multi drug resistant.

shaking incubator (200 oscillations/ min). A volume (less than 900 $\mu \mathrm{L}$ ) of biocide at known concentration was applied to each dilution tube. The final volume of each tube was adjusted to $10.0 \mathrm{ml}$ with the addition of sterile distilled water. The tubes were incubated for $48 \mathrm{~h}$ at $37^{\circ} \mathrm{C}$ and observed for growth at 19 and $48 \mathrm{~h}$. MICs were estimated as the lowest concentration of biocide that inhibited growth.

\section{Induction of biocide adaptation}

2-PE adaptation experiments were performed according to Abdelmalek and Badran (2010). Briefly, fresh E. coli and $P$. aeruginosa cultures were prepared by inoculating $25 \mathrm{~mL}$ of nutrient broth (NB) with $250 \mu \mathrm{L}$ of an overnight culture of the isolates (adjusted to 0.7 optical density(O.D) of A470), and adding an aliquot of $2-\mathrm{PE}$ that is equal to $1 / 4$ of the previously determined MIC. This culture was considered passage 1 (P1). Once the MIC value of this passage was re-determined and density adjusted, $250 \mu \mathrm{L}$ of this culture was inoculated in fresh $25 \mathrm{~mL}$ of N.B media containing $1 / 4$ of the newly determined MIC. This procedure was repeated for several successive passages. The experiments were performed in triplicates, results being the mean of the three MICs determined.

\section{Cross-resistance to antibiotics}

Wild type E. coli ATCC 25922, MDR and 2-PE-adapted MDR E. coli (P2) cultures as well as MDR $P$. aeruginosa and 2-PE- adapted MDR $P$. aeruginosa (P4) were freshly prepared and inoculums size was adjusted to O.D of 0.7 at A470. Antibiotic sensitivity was determined using the disc diffusion method: bacterial cultures were seeded on Mueller Hinton Agar and antibiotic discs were applied; the inhibition zones were measured in millimeters.

\section{Statistical analysis}

Non-parametric Mann-Whitney $U$ test was used to compare MIC values and antibiotic sensitivities of wild type and MDR strains.

\section{RESULTS}

\section{Minimal inhibitory concentrations and adaptation experiments}

Minimal inhibitory concentration of 2-PE was determined for wild type and MDR strains of both $E$. coli and $P$. aeruginosa. MIC of the wild type E. coli ATCC was 4.4 $\mathrm{g} \%$ and of the MDR strain was $4.4 \mathrm{~g} \%$. MDR E. coli was able to grow for two passages only in the presence of sub MIC of 2-PE. A slight increase in MIC of passage 1 was detected. The MIC of 2-PE obtained was $5.3 \mathrm{~g} \%$ that was stable in passage 2 (Table1). On the other hand, the 2PE MIC of MDR $P$. aeuginosa was significantly $(\mathrm{p}<0.05)$ higher $(6.1 \mathrm{~g} \%)$ than 2-PE MIC of the control strain (PAO1) (3.3g \%). MDR $P$. aeruginosa adapted rapidly to the biocide with an almost two fold increase in MIC by passage 4 (P4). The MIC increased significantly $(p<$ 0.05 ) from $6.1 \mathrm{~g} \%$ for the MDR strain to $13.7 \mathrm{~g} \%$ by passage 4 (Table 1). Adaptation experiments could have been pursued for higher values in MIC but since this was not the purpose of this research the authors considered a two fold increase in MIC to be adequate.

\section{Antibiotic sensitivity testing}

The susceptibility of MDR and 2-PE adapted MDR strains of $E$. coli and $P$. aeruginosa for clinically used antibiotics was tested using the disc diffusion method. Results are presented in Tables 2 and 3, respectively. E. coli strain MDR and 2-PE exposed MDR did not show any significant change in antibiotic susceptibility (Table 2), except for both ciprofloxacin and doxycyclin to which the 2-PE exposed MDR strain expressed significant increased sensitivity. However, the situation was different with $P$. aeruginosa. The antibiogram of the MDR strain (Table 3) compared to the antibiogram of MDR 2-PE adapted strain displayed significant change $(p<0.05)$ from resistance to sensitivity for 15 out of 25 tested antibiotics and significant increase $(p<0.05)$ in susceptibility for 6 out of 25 . On the other hand, there was no significant change in susceptibility for both tobramycin and azithromycin, from 13.6 to $20 \mathrm{~mm}$ and from 25 to $18 \mathrm{~mm}$, respectively. Aztreonam stood out with a significant $(p<0.05)$ decrease in the zone of inhibition from 18.6 to $0 \mathrm{~mm}$, being the only case of resistance.

\section{DISCUSSION}

Several reports have indicated that adaptation to some biocides could lead to antibiotic resistance (Braoudaki and Hilton, 2004 ; Carson et al., 2008; Fraise, 2002; Hegstad et al., 2010; Joynson et al., 2002). In a previous work (Abdel Malek and Badran, 2010) adaptation of PAO1 strain of $P$. aeruginosa to 2-PE resulted in increased susceptibility to some antibiotics. A question presented itself in light of these findings; what if clinical MDR isolates were exposed and adapted to 2-PE, would this improve antibiotic susceptibility? This question was investigated in the present study. Results have shown that adaptation to 2-PE was species specific; MDR E. coli was not able to adapt to sub-minimal inhibitory concentrations of 2-PE. A slight increase in 2-PE MIC was observed 
Table 2. Comparison between antibiotic susceptibilities (as measured by zone of inhibition in ( $\mathrm{mm}$ ) of MDR clinical isolate of E.coli and the same isolate after exposure to 2-PE.

\begin{tabular}{|c|c|c|c|}
\hline \multirow{2}{*}{ Antibiotic } & \multicolumn{3}{|c|}{ Zone of inhibition $(\mathrm{mm})( \pm \mathrm{SD})$} \\
\hline & MDR E. coli & 2-PE exposed MDR E. coli & *P-value \\
\hline Gentamicin & $21.6(.05)$ & $21(.1)$ & \\
\hline Amoxacillin & $0(0)$ & $2.6(.46)$ & \\
\hline Doxycyclin & $15.6(.05)$ & $20.6(.46)$ & $<0.05$ \\
\hline Amikacin & $21(.17)$ & $20(0)$ & \\
\hline Rifambicin & $0(0)$ & $0(0)$ & \\
\hline Norfloxacin & $0(0)$ & $0(0)$ & \\
\hline Vancomycin & $0(0)$ & $0(0)$ & \\
\hline Cefotaxime & $0(0)$ & $0(0)$ & \\
\hline Cephalexin & $0(0)$ & $0(0)$ & \\
\hline Ofloxacin & $0(0)$ & $0(0)$ & \\
\hline Chloramphenicol & $21.3(.15)$ & $22.3(.25)$ & \\
\hline Tobramycin & $20.3(.05)$ & $20(0)$ & \\
\hline Azithromycin & $13.6(.05)$ & 13.3(.05) & \\
\hline Sulphamethoxazole & $24.6(.05)$ & $25(.17)$ & \\
\hline Ticar/clav & $7.6(.05)$ & $0(0)$ & \\
\hline Ciprofloxacin & $0(0)$ & $10.3(.11)$ & $<0.05$ \\
\hline Cefixim & $0(0)$ & $0(0)$ & \\
\hline Minocyclin & $17(.1)$ & $21.3(.58)$ & \\
\hline Aztreonam & $15(.1)$ & 13.6(.05) & \\
\hline Ceftizoxime & $0(0)$ & $0(0)$ & \\
\hline Piperacillin & $11.6(.15)$ & $9.6(.05)$ & \\
\hline Ceftazidime & $0(0)$ & $0(0)$ & \\
\hline Ampicillin & $0(0)$ & $0(0)$ & \\
\hline Imipenem & 25.3(.05) & 23(.17) & \\
\hline Nitrofuranton & 20.3(.15) & $20(0)$ & \\
\hline
\end{tabular}

${ }^{*} \mathrm{P}$-value calculated using a non-parametric Mann-Whitney U test.

(Table 1) beyond which the bacteria were not able to adapt or grow. On the other hand, MDR $P$. aeruginosa adapted rapidly to 2-PE with a significant two fold increase in MIC (from 6.1 to $13.7 \mathrm{~g} \%$ ). Such finding is compatible with what has been documented earlier by Bailey et al. (2009) and Braoudaki and Hilton (2004).

When the 2- PE exposed MDR and the MDR E. coli strains were tested for their sensitivity to antibiotics, susceptibility patterns did not vary between the two strains (Table 2). Statistically speaking, we observed significant $(p<0.05)$ increase in susceptibility to ciprofloxa-cin and doxycyclin as seen by increase in the zone of inhibition from 0 to $10 \mathrm{~mm}$ and from 15.6 to $20.6 \mathrm{~mm}$, respectively. However the change in sensitivity is very little and does not mean that 2-PE exposed MDR- E.coli became susceptible, since the minimum zone of inhibition required for these antibiotics are $21 \mathrm{~mm}$ for ciprofloxacin and $16 \mathrm{~mm}$ to doxycycline (Wikler, 2008). Resistance towards hydrophilic fluoroquinolones, (ciprofloxacin, ofloxacin and norfloxacin) in $E$. coli is controlled by the norA gene (Yoshida et al., 1990). Thus, if the suggested efflux pump mediated by the norA gene was inhibited by exposure to 2-PE, this should result in increased sensitivity towards these three floroquinolons. But the effect was only observed towards ciprofloxacin and not the other two floroquinolons. On the other hand, doxycyclin and other antibiotics are substrates to the same efflux pump in MDR $E$. coli isolates (Kern et al., 1994), nevertheless, no change in other antibiotics' sensitivities was observed (Table 2). These results necessitate more investigation.

In comparison, 2- PE adapted MDR $P$. aeruginosa demonstrated a remarkable change in antibiotic susceptibility. Adaptation to 2-PE was accompanied by a significant $(p<0.005)$ complete reversal in antibiotic sensitivity profile from resistance to sensitivity towards 14 out of 25 antibiotics tested. These antibiotics were penicillins (amoxacillin, ampicillin, ticar/clav) cephalosporines (cefotaxime, cephalexin rifampicin, cefixim), quinolones (norfloxacin, ciprofloxacin, ofloxacin,), vancomycin, sulphamethoxazole, nitrofuranton and imipenem. Moreover, 6 out of 25 antibiotics, showed significant $(p<0.005)$ increase in sensitivity and these were tetracycline (doxycyclin, minocycline), gentamycin, piperacillin, ceftazidime and chloramphenicol. On the other hand, sensitivity to aztreonam 
Table 3. Comparison between antibiotic susceptibilities (as measured by zone of inhibition in $\mathrm{mm}$ ) of MDR clinical isolates of $P$. aeruginosa and the same isolate after adaptation to 2-PE.

\begin{tabular}{|c|c|c|c|}
\hline \multirow{2}{*}{ Antibiotic } & \multicolumn{3}{|c|}{ Zone of inhibition $(\mathrm{mm})( \pm \mathrm{SD})$} \\
\hline & MDR P. aeruginosa & 2-PE resistant MDR $P$. aeruginosa & ${ }^{*}$ P-value \\
\hline Gentamicin & $2.3(0.4)$ & $25.3(.4)$ & $p<0.05$ \\
\hline Amoxacillin & $0(0)$ & $36(.6)$ & $p<0.05$ \\
\hline Doxycyclin & $3.3(0.57)$ & $30.3(.35)$ & $p<0.05$ \\
\hline Amikacin & $21.6(0.15)$ & $23.6(.4)$ & ns* \\
\hline Rifambicin & $0(0)$ & $27.6(.61)$ & $p<0.05$ \\
\hline Norfloxacin & $0(0)$ & $28(.6)$ & $p<0.05$ \\
\hline Vancomycin & $0(0)$ & $21.8(.58)$ & $p<0.05$ \\
\hline Cefotaxime & $0(0)$ & 23.6(.55) & $p<0.05$ \\
\hline Cephalexin & $0(0)$ & $29(.45)$ & $p<0.05$ \\
\hline Ofoxacin & $0(0)$ & $24(.17)$ & $p<0.05$ \\
\hline Chloramphenicol & $2.3(.40)$ & $25(.05)$ & $p<0.05$ \\
\hline Tobramycin & $13.6(.46)$ & $20(.88)$ & ns \\
\hline Azithromycin & $25(.26)$ & $18.3(.47)$ & ns \\
\hline Sulphamethoxazole & $0(0)$ & $27.2(.17)$ & $p<0.05$ \\
\hline Ticar/clav & $0(0)$ & $35.3(.66)$ & $p<0.05$ \\
\hline Ciprofloxacin & $0(0)$ & 28.3(.60) & $p<0.05$ \\
\hline Cefixim & $0(0)$ & $15(1.21)$ & $p<0.05$ \\
\hline Minocyclin & $9.6(.46)$ & $27(.60)$ & $p<0.05$ \\
\hline Aztreonam & 18.6(.15) & $0(0)$ & $p<0.05$ \\
\hline Ceftizoxime & 13.3(1.15) & 28(.39) & ns \\
\hline Piperacillin & $17(.78)$ & $34(.85)$ & $p<0.05$ \\
\hline Ceftazidime & $15(.1)$ & $19.3(.84)$ & $p<0.05$ \\
\hline Ampicillin & $0(0)$ & $34.3(.40)$ & $p<0.05$ \\
\hline Imipenem & $0(0)$ & 39.5(.35) & $p<0.05$ \\
\hline Nitrofuranton & $0(0)$ & $21(.1)$ & $p<0.05$ \\
\hline
\end{tabular}

${ }^{*} \mathrm{P}$ value calculated using a non-parametric Mann-Whitney $U$ test; *ns: not significant.

was completely lost $(p<0.005)$ (Table3). Such alterations in antibiotic sensitivity have been documented previously (Joynson et al., 2002), though MDR isolates were not the focus of such studies.

2-PE adaptation by P.aeruginosa PAO1 was accompanied by an increased sensitivity towards the antibiotic aztreonam (Abdel Malek and Badran, 2010). Interestingly though, adaptation of MDR $P$. aeruginosa isolates to 2$\mathrm{PE}$ resulted in aztreonam resistance. Aztreonam resistance is known to be mainly due to increased mexA expression (Walsh et al., 2003). However, Abdelmalek and Badran (2010) found that the antibiotic susceptibility changes in the 2-PE resistant $P$. aeruginosa PAO1 are independent of RND efflux pumps. Similarly, we can say that the gain of the aztreonam resistant phenotype in our case might not be due to over expression of mex $A$. This poses yet another question into the complex arena of biocide/antibiotic cross resistance. Two very clinically importantand useful antipseudomonal antibiotics, imipenem and gentamycin were not affected by 2-PE adaptation in the PAO1 strain (Abdel Malek and Badran, 2010) however, MDR $P$. aeruginosa gained full sensitivity to these two antibiotics after the adaptation to 2-PE in this study (Table 3). The 2-PE adaptation of MDR P.aeruginosa strain was shown to manifest differently than the laboratory strain PAO1 $P$. aeruginosa. Although both involved increased susceptibility to antibiotics and reduced to others but the response to aztreonam and imipenem was different in both strains.

Imipenem resistance is known to be due to suppression of OprD thus regaining sensitivity to imipenem by the MDR 2-PE strain and the loss of aztreonam susceptibility further supports our hypothesis that the reason behind these changes is mainly outer membrane alterations. 2PE has been reported to induce outer membrane changes (Abdel Malek and Badran, 2010; Denyer, 1995) which according to our results varies between species. MDR isolates of $P$. aeruginosa are known to overexpress and express multiple efflux pumps, as well as undergo changes in outer membrane structure (Toma's et al., 2010) thus the effect of 2-PE will eventually express a different phenotype than the wild type PAO1.

Does antibiotic resistance bring about biocide resistance? A question raised by Stickler (2002) who examined 
clinical isolates that were chlorhexidine resistant as well as antibiotic resistant from patients suffering from urinary tract infection (UTI). The author explained that the emergence of such phenotype is due to excessive use of chlorhexidine in catheter management. In the present study, clinical MDR strain of $P$. aeruginosa had a significantly $(p<0.05)$ elevated MIC towards $2-P E$ in comparison to the PAO1 strain (Table 1) although the MDR clinical strain was not exposed to 2-PE like in the case of chlorhexidine. Thus, there has been a low level of resistance towards this biocide that accompanied the MDR status that we would loosely say it came with the antibiotic resistance. The present research is still in the pursuit of understanding the relationship between biocide misuse and antibiotic resistance. Results obtained in this research draw the attention to a different, rather interesting, effect that can be imposed on antibiotics susceptibility as a result of biocide resistance. These findings suggest further questions that need more investigation. For example, what exactly in 2-PE adaptation triggered this effect? Is this effect on multidrug resistance uniform for other biocides? We believe that these findings can open up a new window in the future for the treatment or prevention of multidrug resistant nosocomial infections.

\section{REFERENCES}

Abdel Malek SM, Al-Adham IS, Matalka KZ, Collier PJ (2009). Pseudomonas aeruginosa PAO1 resistance to zinc pyrithione: phenotypic changes suggest the involvement of efflux pumps. Curr Microbio. 59: 95-100.

Abdel Malek SMA, Badran YR (2010). Pseudomonas aeruginosa PAO1 adapted to 2-phenoxyethanol shows cross-resistance to dissimilar biocides and increased susceptibility to antibiotics. Folia Microbio. 55: 588-592.

Aiello AE, Marshall B, Levy SB, Della-Latta P, Lin SX, Larson E (2005). Antibacterial cleaning products and drug resistance. Emerg Infect. Dis. 11(10): 1565-1570.

Aiello AE, Larson EL, Levy SB (2007). Consumer antibacterial soaps: effective or just risky? Clin. Infect. Dis. 45 (S2): S137-147.

Bailey AM, Constantinidou C, Ivens A, Garvey MI, Webber MA, Coldham N, Hobman JL, Wain J, Woodward MJ, Piddock LJV (2009). Exposure of Escherichia coli and Salmonella enterica serovar Typhimurium to triclosan induces a species-specific response, including drug detoxification. J. Antimicrob. Chemother. 64 (5): 973985.

Beveridge EG, Boyd I, Dew I, Haswell M, Lowe CWG (1991). Electron and light microscopy of damaged bacteria. Society for Applied Bacteriology. Technical Series. 27: 135-153.

Bloomfield SF (1991). Methods for assessing antimicrobial activity. In: Denyer SP, Hugo WB (eds). Mechanisms of Action of Chemical Biocides: Their Study and Exploitation. Blackwell Scientific Publications, Oxford, UK, pp 1-22.

Braoudaki M, Hilton AC (2004). Adaptive resistance to biocides in Salmonella enterica and Escherichia coli $\mathrm{O} 157$ and cross-resistance to antimicrobial agents. J. Clin. Mic. 42: 73-78.

Carson RT, Larson E, Levy SB, Marshall BM, Aiello AE (2008). Use of antibacterial consumer products containing quaternary ammonium compounds and drug resistance in the community. J. Antimicrob. Chemother. 62:1160-1162.

Denyer SP (1995). Mechanisms of action of antibacterial biocides. International biodeterioration \& biodegradation, 36 (3-4): 227-245.

Fraise AP (2002). Biocide abuse and antimicrobial resistance- a cause for concern? J. Antimicrob. Chemother. 49:11-12.
Hegstad K S, Langsrud BT, Lunestad AA, Scheie M, Sunde SP, Yazdankhah (2010). Does the wide use of quaternary ammonium compounds enhance the selection and spread of antimicrobial resistance and thus threaten our health? Microb. Drug Resist. 16: 91-104.

Hubner NO, Siebert J, Kramer A (2010). Octenidine dihydrochloride, a modern antiseptic for skin, mucous membranes and wounds. Skin Pharmacol. Physiol. 23: 244-258.

Jones MV, Herd T M, Christie H J (1989). Resistance of Pseudomonas aeruginosa to amphoteric and quaternary ammonium biocides. Microbios. 58 (234): 49-61.

Joynson JA, Forbes B, Lambert RJW (2002). Adaptive resistance to benzalkonium chloride, amikacin and tobramycin: the effect on susceptibility to other antimicrobials. J. Appl. Microb. 93: 96-107.

Karatzas KAG, Webber MA, Jorgensen F, Woodward MJ, Piddock LJV, Humphrey TJ (2007). Prolonged treatment of Salmonella enterica serovar Typhimurium with commercial disinfectants selects for multiple antibiotic resistance, increased efflux and reduced invasiveness. J. Antimicrob. Chemother. 60: 947-955.

Kern WV, Andriof E, Oethinger M, Kern P, Hacker J, Marre R (1994). Emergence of fluoroquinolone-resistant Escherichia coli at a cancer center. Antimicrob Agents and Chemother. 38: 681-687.

Loughlin MF, Jones MV, Lambert PA (2002). Pseudomonas aeruginosa cells adapted to benzalkonium chloride show resistance to other membrane-active agents but not to clinically relevant antibiotics. $J$ Antimicrob. Chemother. 49: 631-639.

Maillard JY (2010). Emergence of bacterial resistance to microbicides and antibiotics. Microbiol. Aust. 31(4): 159-164.

Poole K (2012). Bacterial stress responses as determinants of antimicrobial resistance. J. Antimicrob. Chemother. doi: 10.1093/jac /dks 196

Russell AD (2003). Similarities and differences in the responses of microorganisms to biocides. J Antimicrob Chemother. 52:750-763.

Stickler DJ (2002). Susceptibility of antibiotic-resistant Gram-negative bacteria to biocides: a perspective from the study of catheter biofilms. J. Appl. Microbio. 92:163S-170S.

Strateva T, Yordanov D (2009). Pseudomonas aeruginosa-a phenomenon of bacterial resistance. J Med Microbio. 58:1133-1148.

Toma's M, Doumith MM, Warner JF, Turton A, Beceiro G, Bou DM, Livermore N, Woodford (2010). Efflux pumps, OprD porin, AmpC Blactamase, and multiresistance in Pseudomonas aeruginosa isolates from cystic fibrosis patients. Antimicrob. Agents Chemother . 54: 2219-2224.

Walsh SE, Maillard JY, Russell AD, Catrenich CE, Charbonneau DL, Bartolo RG (2003). Development of bacterial resistance to several biocides and effects on antibiotic susceptibility. J. Hosp. Infect. 55: 98-107.

Wikler, MA (2008). Performance standards for antimicrobial disk susceptibility tests: eighteenth informational supplement. 18. ed. Wayne, PA: Clinical and Laboratory Standards Institute. p.181 CLSI document M100-S18). ISBN 1562386530.

Wilson M, Stanley A, Bansal G, Newman HN (1990). Effect of phenoxyethanol, chlorhexidine and their combination on subgingival plaque bacteria. J. Antimicrob. Chemother. 25: 921-929.

Winder CL, Al-Adham, ISI, Abdel Malek SMA, Buultjens TEJ, Horrocks AJ, Collier PJ (2000). Outer membrane protein shifts in biocideresistant Pseudomonas aeruginosa PAO1. J. Appl. Microbiol., 89: 289-295.

Yoshida H, Bogaki M , Nakamura S, Ubukata K, Konno M (1990). Nucleotide sequence and characterization of the Staphylococcus aureus norA gene, which confers resistance to quinolones. J. Bacterio. 172: 6942-6949. 